University of Nebraska - Lincoln

DigitalCommons@University of Nebraska - Lincoln

3-1-1999

Formation free energy of clusters in vapor-liquid nucleation: $A$ Monte Carlo simulation study

K.J. Oh

University of Nebraska-Lincoln

Xiao Cheng Zeng

University of Nebraska-Lincoln, xzeng1@unl.edu

Follow this and additional works at: https://digitalcommons.unl.edu/chemzeng

Part of the Chemistry Commons

Oh, K.J. and Zeng, Xiao Cheng, "Formation free energy of clusters in vapor-liquid nucleation: A Monte Carlo simulation study" (1999). Xiao Cheng Zeng Publications. 61.

https://digitalcommons.unl.edu/chemzeng/61

This Article is brought to you for free and open access by the Published Research - Department of Chemistry at DigitalCommons@University of Nebraska - Lincoln. It has been accepted for inclusion in Xiao Cheng Zeng Publications by an authorized administrator of DigitalCommons@University of Nebraska - Lincoln. 


\title{
Formation free energy of clusters in vapor-liquid nucleation: A Monte Carlo simulation study
}

\author{
K. J. Oh and X. C. Zeng ${ }^{\text {a) }}$ \\ Department of Chemistry, University of Nebraska-Lincoln, Lincoln, Nebraska 68588
}

(Received 26 October 1998; accepted 24 November 1998)

\begin{abstract}
The formation free energy of clusters in a supersaturated vapor is obtained by a constrained Monte Carlo technique. A key feature of this approach is to set an upper limit to the size of cluster. This maximum cluster size serves essentially as an extra thermodynamic variable that constrains the system. As a result, clusters larger than the critical cluster of nucleation in the supersaturated vapor can no longer grow beyond the limiting size. Like changing the overall density of the system, changing the maximum cluster size also results in a different supersaturation and thereby a different formation free energy. However, at the same supersaturation and temperature it is found that the formation free energy has a unique value, independent of the upper limit of cluster size. The predicted size of critical cluster of nucleation is found to be consistent with the nucleation theorem as well as previous results using different simulation approaches. (c) 1999 American Institute of Physics. [S0021-9606(99)51009-X]
\end{abstract}

\section{INTRODUCTION}

The formation free energy $\Delta G_{i}$ for a cluster of size $i$ in a supersaturated vapor is crucial to the determination of the steady-state nucleation rate. ${ }^{1}$ In the classical nucleation theory, ${ }^{2,3}$ the cluster is assumed to be a macroscopic drop of liquid; $\Delta G_{i}$ of the cluster is obtained using properties of the bulk fluid. The classical nucleation theory has been widely used to correlate experimental measurements. ${ }^{4,5}$ It has been shown that the theory generally predicts the supersaturation dependence of the nucleation rate quite well but not the temperature dependence. To develop a better theory, either phenomenological or molecular, of nucleation, it would be desirable to obtain the formation free energy (i.e., barrier of nucleation) for a commonly studied model system, such as the Lennard-Jones $(12,6)$ fluid, from first principles. To this end, here we present a Monte Carlo simulation method to determine the equilibrium distribution of cluster sizes in a supersaturated vapor. By appealing to the principle of detailed balance, $\Delta G_{i}$ can be obtained straightforwardly. ${ }^{6}$

The equilibrium distribution of clusters $N_{i}$ in a supersaturated vapor has a simple relation with $\Delta G_{i}$ as follows:

$$
N_{i}=N_{1} e^{-\Delta G_{i} / k_{B} T},
$$

where $N_{1}$ is the number of monomers, $k_{B}$ is Boltzmann constant, and $\Delta G_{i}$ is the difference in the Gibbs free energy between the $i$-mer and the monomer at the pressure of the supersaturated vapor at temperature $T$. This equation has been derived previously ${ }^{3,7-10}$ and used to infer the formation free energy from the equilibrium distribution of clusters. Here we determine $N_{i}$ by Monte Carlo simulation and then compute $\Delta G_{i}$ from Eq. (1).

There are known obstacles to the computer simulation of supersaturated systems. First, because the supersaturated vapor is metastable, once a cluster with a size larger than the critical cluster of nucleation forms, it will keep growing. If

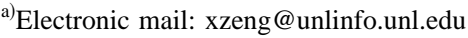

the system is open, the cluster will eventually become a macroscopic liquid phase. Second, because the critical cluster is in unstable equilibrium with respect to the vapor (resulting in a minimum in $N_{i}$ or a maximum in $\Delta G_{i}$ as a function of $i$ ), long computation times may be needed to achieve good statistics of $N_{i}$, particularly for $i$ close to the critical value. Third, the periodic boundary condition and finite system size may also have large effects on the rate of nucleation. For example, in a simulation study of the onset of crystallization Swope and Andersen ${ }^{11}$ found that the system size effects become unimportant only when the system contains more than $10^{4}$ molecules.

To circumvent the first problem Corti and Debenedetti ${ }^{12}$ applied the idea of constrained equilibrium of Reiss, ${ }^{13,14}$ that is, the rigorous statistical mechanics of metastable equilibrium is essentially the statistical mechanics of a constrained system. Corti and Debenedetti ${ }^{12}$ divided the computational cell into a number of subcells of the same size and limited the number of molecules in each subcell. Thus, the system is constrained to be homogeneous, even though it is metastable. Corti and Debenedetti, ${ }^{12}$ however, found a dependence of nucleation barrier on the system size (the third problem mentioned above). This dependence is presumably due to the relatively small system sizes (with 108 and 256 particles) used in the simulation.

In the present work, we also adopted the idea of constrained equilibrium, although we take a different approach. Motivated from conventional statistical mechanical treatments of the supersaturated vapor, ${ }^{3,7}$ where the cluster distribution is generally truncated at a certain size, we chose an upper limit of cluster size $i_{\max }$ such that $i_{\max }$ is larger than the critical cluster size $i^{*}$. Thus, in the simulation, clusters larger than $i^{*}$ cannot grow larger than $i_{\text {max }}$. A special feature of this approach is that at a given temperature $T$ and overall density $N / V$ a fixed value of $i_{\max }$ leads to a unique equilibrium cluster distribution and henceforth a unique supersaturation. Here $N$ is the number of molecules in the system and 


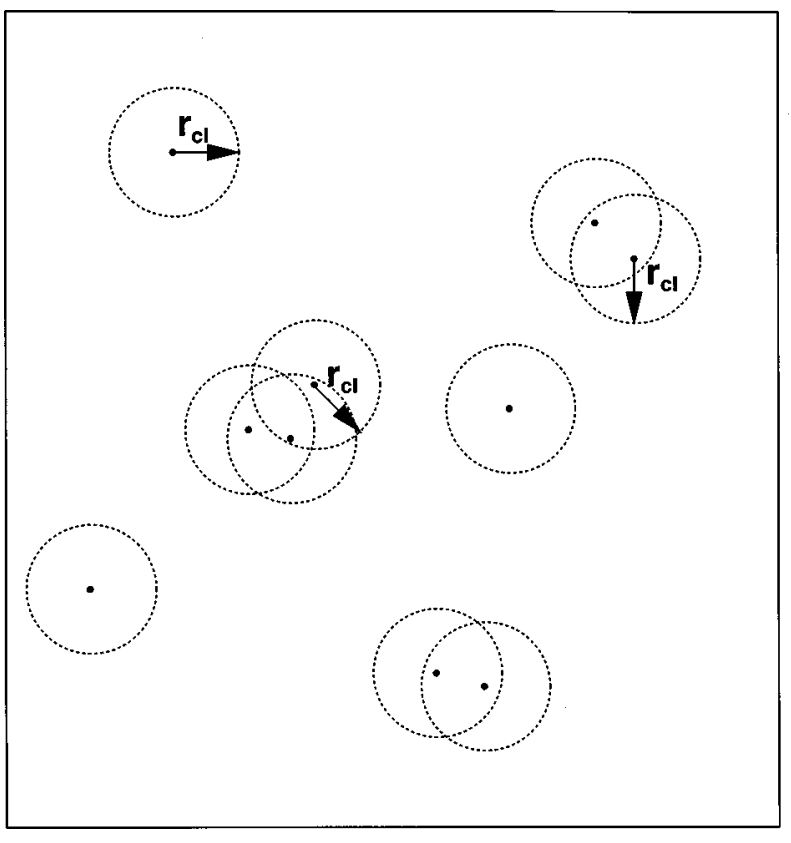

FIG. 1. Schematic illustration of the Stillinger clusters. Here three monomers, two dimers and one trimer are shown.

$V$ is the system volume. In contrast to the conventional canonical system, here four independent thermodynamic variables, $N, V, T$, and $i_{\max }$ are needed to describe the constrained system.

\section{MONTE CARLO SIMULATION}

The system chosen in this study is the Lennard-Jones $(12,6)$ fluid, for which the pairwise intermolecular potential is given by

$$
u(r)=4 \epsilon\left[(\sigma / r)^{12}-(\sigma / r)^{6}\right],
$$

where $r$ is the intermolecular distance. The molecular parameters in Eq. (2) are those of argon, $\epsilon / k=119.8 \mathrm{~K}$ and $\sigma$ $=3.405 \AA$. In all simulations, the potential is truncated at $4.5 \sigma$. The cell-linked list method ${ }^{15}$ was used to speed up the simulation.

Monte Carlo simulation was performed at fixed $N, V, T$, and $i_{\max }$ with the usual periodic boundary conditions. Configurations of the supersaturated vapor were generated by conventional Metropolis algorithm. After each Monte Carlo trial move, clusters are identified according to the Stillinger's criterion. ${ }^{16}$ Once a cluster is found larger than $i_{\max }$, that Monte Carlo trial move is rejected. According to Stillinger's criterion, any two molecules are connected if the intermolecular distance is less than a cutoff distance $r_{c l}$, which is set to $1.5 \sigma$ (see Fig. 1). This value of the $r_{c l}$ has been used in several recent studies. ${ }^{9,10}$ In particular, Reiss and co-workers ${ }^{10}$ obtained $r_{c l}$ dependence of $n / v$-Stillinger cluster lifetime for the Lennard-Jones system and found that the lifetime increases rapidly with $r_{c l}$ until $r_{c l}$ reaches about $1.5 \sigma$ and enters a plateau corresponding to a roughly constant lifetime. Reiss and co-workers argued that it is advisable to choose a value of $r_{c l}$ within this plateau where the lifetime is long. A value of $1.5 \sigma$ for $r_{c l}$ corresponds to the onset of the plateau.

The main advantage of Stillinger's criterion is that it allows us to avoid redundant counting of clusters. ${ }^{6,17}$ Abraham and Barker ${ }^{18}$ pointed out that under Stillinger's criterion it could occur that two compact clusters are viewed as one, even though the two clusters are connected by only one molecule. However, this situation is extremely rare at the low vapor densities pertinent to nucleation under normal circumstances. Here, the compact clusters are the most probable.

Monte Carlo simulations were carried out under the several different conditions $\left(N / V\right.$ and $\left.i_{\max }\right)$ listed in Table I. The configuration was initially a face-centered-cubic (fcc) lattice having an overall density $N / V$ larger than $\rho_{\text {sat }}$, where $\rho_{\text {sat }}$ is the vapor density at saturation. Note that the larger of $N / V \rho_{\text {sat }}$, the faster the system reaches the constrained equilibrium state. The mean number of cluster of size $i,\left\langle N_{i}\right\rangle$, is obtained by averaging over many configurations, where $N_{i}$ satisfies

$$
\sum_{i=1}^{i_{\max }} i N_{i}=N
$$

A good measure of the supersaturation $S$ is given by

$$
S=\sum_{i=1}^{i_{\max }} \rho_{i} / \rho_{\mathrm{sat}},
$$

\begin{tabular}{|c|c|c|c|c|c|c|c|c|}
\hline$T$ & $N$ & $N / V$ & $i_{\max }$ & equi $^{\mathrm{a}}$ & $a^{b} e^{b}$ & $S$ & $i^{* c}$ & $\Delta G_{i}^{*} / k_{B} T^{\mathrm{c}}$ \\
\hline \multirow[t]{8}{*}{0.67} & 4000 & 0.023 & 30 & $10^{8}$ & $10^{8}$ & 9.37 & 20 & 8.21 \\
\hline & & & 40 & & & 8.82 & 23 & 9.17 \\
\hline & & & 50 & & & 8.04 & 27 & 10.83 \\
\hline & 8788 & & 30 & & & 9.39 & 21 & 8.24 \\
\hline & & & 40 & & & 8.78 & 23 & 9.28 \\
\hline & & & 50 & & & 8.04 & 26 & 10.78 \\
\hline & 4000 & 0.03 & 40 & & & 9.05 & & \\
\hline & & 0.04 & & & & 9.25 & & \\
\hline 0.71 & 4000 & 0.02 & 90 & $10^{8}$ & $10^{9}$ & 5.33 & 44 & 16.73 \\
\hline
\end{tabular}

TABLE I. Calculated supersaturation $S$, the size of critical cluster $i^{*}$, and the formation free energy of the critical cluster $\Delta G_{i}^{*} / k_{B} T . T$ is in unit of $\epsilon / k_{B}$ and $N / V$ is in units of $\sigma^{-3}$.

${ }^{a}$ Number of Monte Carlo steps for equilibration.

${ }^{\mathrm{b}}$ Number of Monte Carlo steps for averaging.

${ }^{c}$ From polynomial fit of degree six. 


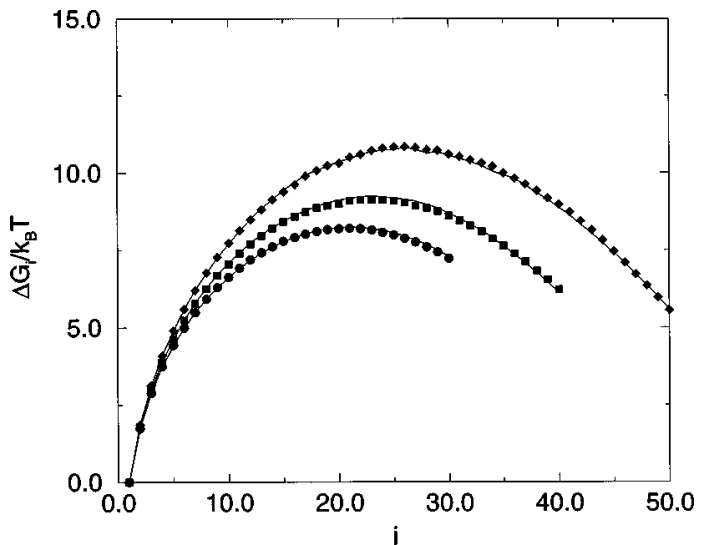

FIG. 2. The $\Delta G_{i} / k_{B} T$ as a function of $i$ for $N=4000$ (filled symbols) and 8788 (solid lines) under the condition $T=0.67 \epsilon / k_{B}, N / V=0.023 \sigma^{-3}$, and $i_{\max }=30$ (circles), 40 (squares), and 50 (diamonds).

where $\rho_{i}=\left\langle N_{i}\right\rangle / V$. For the Lennard-Jones system, $\rho_{\text {sat }}$ $=0.00161$ and $0.0024 \sigma^{-3}$ at temperature $T=0.67 \epsilon / k_{B}$ and $0.71 \epsilon / k_{B}$, respectively. ${ }^{19}$

\section{RESULTS AND DISCUSSION}

The principal results of all simulations are given in Table I, where the critical cluster size $i^{*}$ and the corresponding formation free energy $\Delta G_{i}^{*}$ are obtained from fitting $\Delta G_{i}$ versus $i$ data via a polynomial of degree six.

\section{A. $N / V$ and $i_{\max }$ dependence}

Figure 2 shows $\Delta G_{i}$ as a function of Stillinger cluster size $i$ for two $N$ (4000 and 8788) and three $i_{\max }$ (30,40, and 50 ), all at the same temperature $T=0.67 \epsilon / k_{B}$ and overall density $N / V=0.023 \sigma^{-3}$. Figure 3 shows the calculated critical cluster size $i^{*}$, the formation free energy of critical cluster $\Delta G_{i}^{*}$, and supersaturation $S$ for three $i_{\max }$. As can be seen in Fig. 3(c), the supersaturation $S$ decreases with increasing $i_{\max }$ at the given temperature and overall density. This is because a larger value of $i_{\max }$ results in formation of

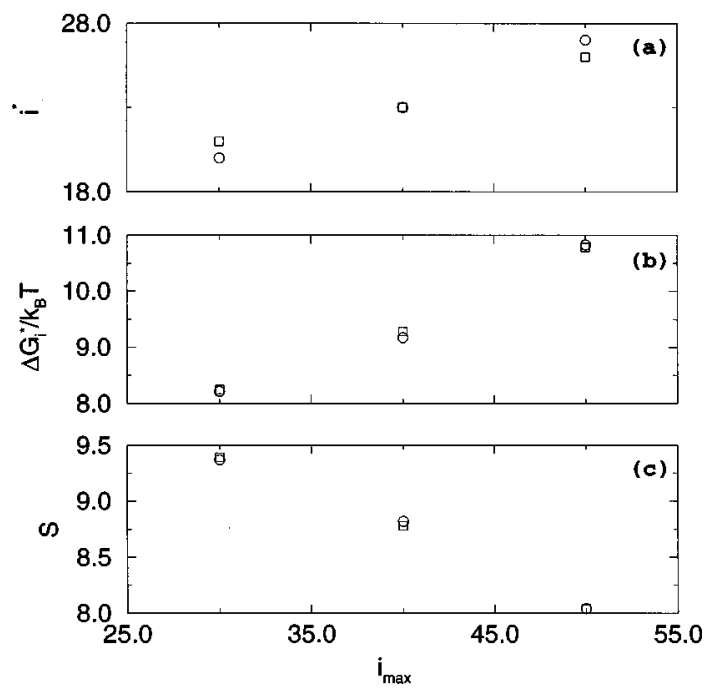

FIG. 3. The calculated $i^{*}, \Delta G_{i}^{*} / k_{B} T$ and $S$ as a function $i_{\max }$ from curves in Fig. 2. Circles and squares represent data for $N=4000$ and 8788 , respectively.

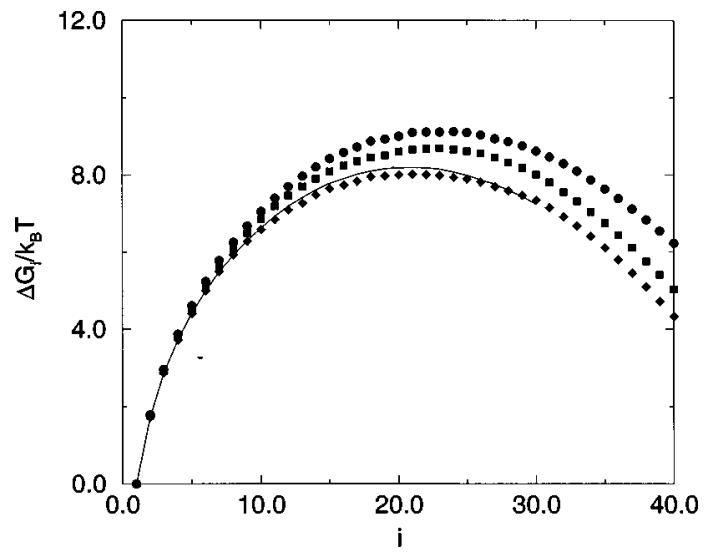

FIG. 4. The $\Delta G_{i} / k_{B} T$ as a function of $i$ for $N=4000$ under the condition $T=0.67 \epsilon / k_{B}, i_{\max }=40$, and $N / V=0.023$ (circles), 0.03 (squares), and $0.04 \sigma^{-3}$ (diamonds). The solid line represents data obtained under the condition $i_{\max }=30$ and $N / V=0.023 \sigma^{-3}$, for the same $N$ and $T$.

more clusters and thus more depletion of monomers. Indeed, increasing $i_{\max }$ is effectively analogous to enlarging the volume of system $V$, both lead to a smaller supersaturation (though different constrained equilibrium states). In other words, as $V, i_{\max }$ should be also viewed as an independent variable to describe the supersaturated state in the constrained equilibrium. ${ }^{14}$ As shown in Figs. 3(a) and 3(b), a smaller value of $S$ corresponds to a larger critical cluster size $i^{*}$ and a higher formation free energy $\Delta G_{i}^{*}$.

A practical limitation in choosing $i_{\max }$ arbitrarily large is the resulting poor statistics. For instance, for $i_{\max }=100$, we did not obtain a smooth $\Delta G_{i}$ curve for $N=4000, T$ $=0.67 \epsilon / k_{B}$, and $N / V=0.023 \sigma^{-3}$, even after $10^{8}$ Monte Carlo steps. On the other hand, $i_{\max }$ cannot be chosen too small, since $i_{\max }$ must be larger than $i^{*}$ in order to infer the properties of critical cluster. For instance, we cannot observe the critical cluster under the condition $N=4000, T$ $=0.67 \epsilon / k_{B}$, and $N / V=0.023 \sigma^{-3}$ if $i_{\max }=20$.

Figure 4 shows $\Delta G_{i}$ as a function of $i$ for three different overall densities $N / V=0.023,0.03$, and $0.04 \sigma^{-3}$, all under the condition $N=4000, T=0.67 \epsilon / k_{B}$, and $i_{\max }=40$. Figure 5 shows plots of $i^{*}, \Delta G_{i}^{*}$, and $S$ as a function $i_{\max }$. As expected, the supersaturation $S$ increases as the overall density $N / V$ is increased.

In summary, there are two routes to control the supersaturation of vapor in the simulation. One is changing $i_{\max }$ and the other is changing $N / V$. We examined the selfconsistency of the approach by checking whether the $\Delta G_{i}$ curves, obtained separately from each route, overlap with each other when both the supersaturation and temperature are fixed. To this end, we compared two curves in Fig. 4: the solid curve which corresponds to the condition $N=4000, T$ $=0.67 \epsilon / k_{B}, N / V=0.023 \sigma^{-3}$, and $i_{\max }=30$ and the filled diamond curve which corresponds to the condition $N$ $=4000, T=0.67 \epsilon / k_{B}, N / V=0.04 \sigma^{-3}$, and $i_{\max }=40$. Although the two curves are obtained under quite different values of $N / V$ and $i_{\max }$, both curves nearly overlap with each other, thereby giving almost the same size of critical cluster $i^{*}$ and free energy of formation $\Delta G^{*}$ [see Figs. 5(a) and 

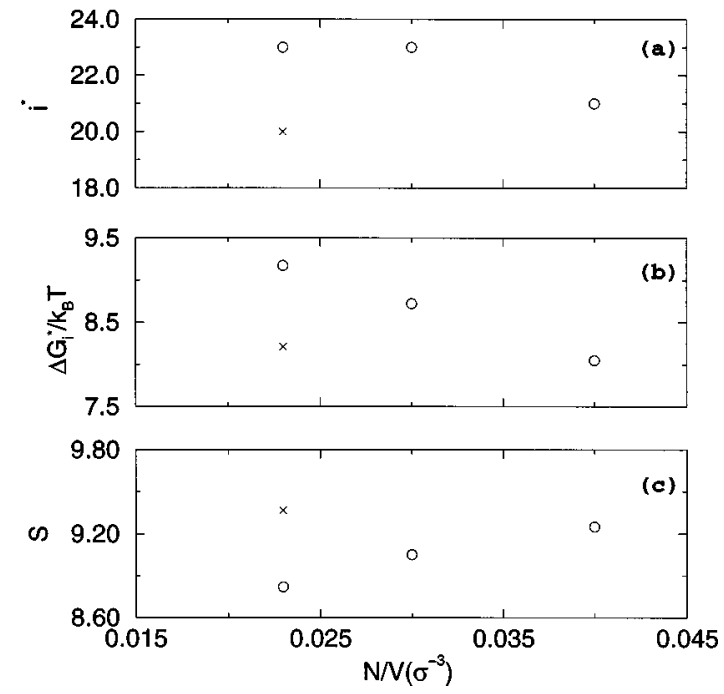

FIG. 5. The calculated $i^{*}, \Delta G_{i}^{*} / k_{B} T$, and $S$ as a function of $N / V$ from curves in Fig. 4. The cross is calculated from the solid line in Fig. 4.

$5(\mathrm{~b})]$. This is indeed expected because the supersaturation in both cases is nearly the same [see Fig. 5(c)].

In this work, the obtained critical cluster sizes are typically within the range of 20-50. As discussed above, the present approach is not computationally the most efficient to study vapors at relatively low supersaturation under which large critical cluster sizes (e.g., 100) are involved. To sample large critical clusters one has to choose a larger $i_{\max }$ in conjunction with a larger system size and longer Monte Carlo runs. In this sense, our approach complements the umbrellasampling approach by ten Wolde et al., ${ }^{9,20}$ which was designed particularly to study vapors at relatively low supersaturations. For the Lennard-Jones system, the highest supersaturation they considered was 2.2 and the size of critical cluster studied was as large as 300 .

\section{B. System size dependence}

As shown in Figs. 3(a)-3(c), we obtained nearly the same results of $i^{*}, \Delta G_{i}^{*}$, and $S$, using two system sizes $N$ $=4000$ (circles) and 8788 (squares). Thus, for this choice of system sizes the finite-size effect (the third problem mentioned in Sec. I) is quite small.

In Fig. 6 we show a snapshot for the simulation under the condition $N=4000, T=0.67 \epsilon / k_{B}, N / V=0.03 \sigma^{-3}$, and

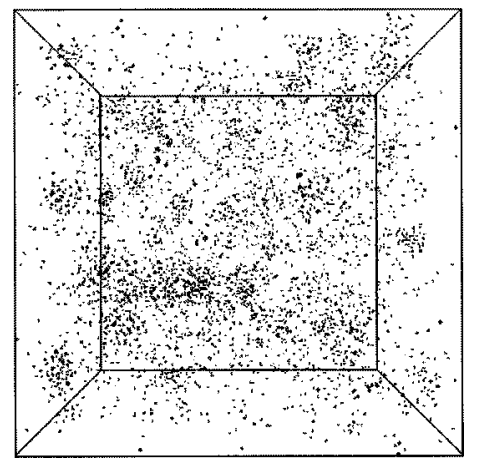

FIG. 6. A snapshot from simulation under the condition $N=4000, T$ $=0.67 \epsilon / k_{B}, N / V=0.03 \sigma^{-3}$, and $i_{\max }=40$.

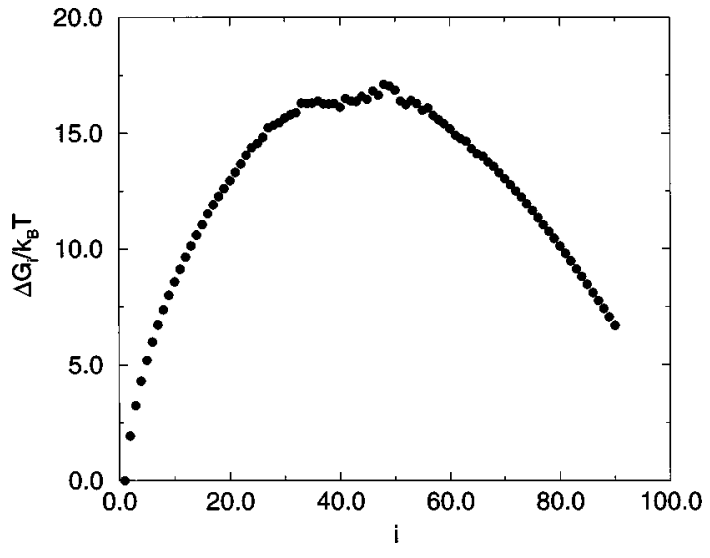

FIG. 7. The $\Delta G_{i} / k_{B} T$ as a function of $i$ under the condition $N=4000, T$ $=0.71 \epsilon / k_{B}, N / V=0.02 \sigma^{-3}$, and $i_{\max }=90$.

$i_{\max }=40$. It can be seen that the system contains a large number of smaller clusters because of the size constraint $i_{\max }$. The presence of such a large number of small clusters in constrained equilibrium allows us to achieve better statistics of the critical cluster. Without using the constraint $i_{\max }$, we would observe a big cluster ${ }^{21,22}$ in equilibrium with the surrounding vapor. In the canonical ensemble, the size of this cluster is limited by the total number of molecules $N$. However, the size of cluster would be comparable to $N$ and as a result, good statistics would not be attained for the critical cluster.

\section{Comparison with other simulations}

Recently, Zhukhovitskii ${ }^{23}$ developed an efficient simulation method to predict the size of critical cluster $i^{*}$. For the Lennard-Jones $(12,6)$ fluid, he obtained $i^{*}$ as a function of supersaturation at $T=0.7017 \epsilon / k_{B}$. For example, Zhukhovitskii found $i^{*} \sim 68$ for supersaturation $S=4.4$. By using a quite different simulation approach, Reiss and co-workers ${ }^{10}$ found $i^{*} \sim 70$ at almost the same supersaturation and temperature.

Zhukhovitskii also obtained $i^{*} \sim 40$ for $S=5.3$ at $T$ $=0.7017 \epsilon / k_{B}$. Our results at temperature $T=0.71 \epsilon / k_{B}$ are shown in Fig. 7, where $\Delta G_{i}$ is plotted as a function of $i$ under the condition $N=4000, N / V=0.02 \sigma^{-3}$, and $i_{\max }$ =90. A billion Monte Carlo steps were used to obtain a relatively smooth curve. Numerical results are listed in Table I. We found the size of critical cluster $i^{*} \sim 44$ for $S=5.33$, which is quite close to that of Zhukhovitskii.

\section{Comparison with the classical nucleation theory}

In the classical nucleation theory, ${ }^{2}$ properties of critical nucleus $i^{*}$ and $\Delta G_{i}^{*}$ are given by

$$
i^{*}=\frac{32 \pi \gamma^{3}}{3\left(k_{B} T \ln S\right)^{3} \rho_{l}^{2}}
$$

and

$$
\Delta G_{i}^{*}=\frac{16 \pi \gamma^{3}}{3\left(k_{B} T \rho_{l} \ln S\right)^{2}},
$$



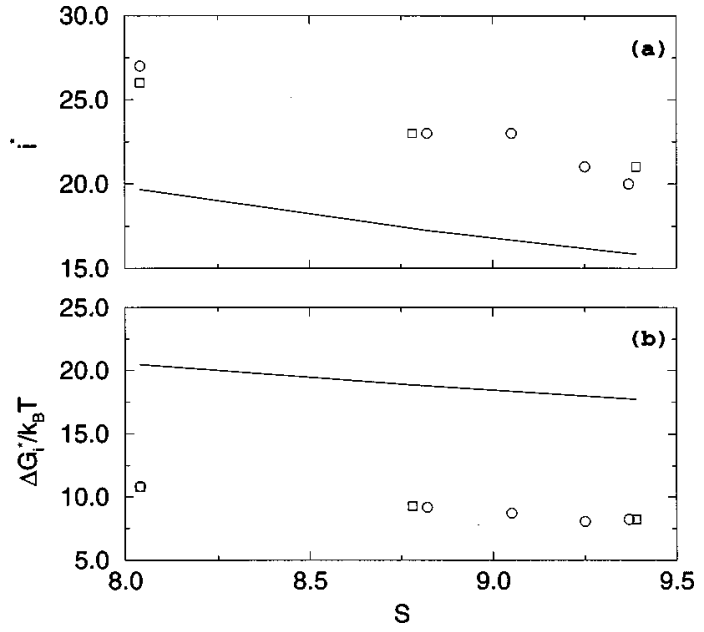

FIG. 8. The $i^{*}$ and $\Delta G_{i}^{*} / k_{B} T$ as a function of $S$ at $T=0.67 \epsilon / k_{B}$. The solid line represents the results from the classical nucleation theory.

where $\gamma$ is planar surface tension, $\rho_{l}$ is the liquid density at saturation. Here, for Lennard-Jones system, $\gamma=1.03 \epsilon / \sigma^{2}$ and $\rho_{l}=0.82 \sigma^{-3}$ at $T=0.67 \epsilon / k_{B}$.

Figures 8(a) and 8(b) show results of $i^{*}$ and $\Delta G_{i}^{*}$ together with results of the classical nucleation theory (CNT) as a function of supersaturation at $T=0.67 \epsilon / k_{B}$. Clearly, CNT overestimates the formation free energy of the critical cluster and underestimates the size. This conclusion is consistent with an earlier prediction from density functional theory of nucleation. ${ }^{24}$

Similar conclusions have also been drawn from experiments. For example, in a study of $n$-nonane nucleation ${ }^{25}$ Katz and co-workers found that CNT predicts the dependence of the nucleation rate on supersaturation rather well, but not the dependence on $T$. The predicted rates can be eight orders of magnitude too small at low temperatures, which indicates that CNT overestimates the formation free energy of the critical cluster of $n$-nonane at low temperatures.

In passing, we note that McGraw and Laaksonen ${ }^{26}$ recently proposed several scaling relations for the size of critical nucleus, the formation free energy of the critical nucleus $\Delta G^{*}$ and their combination. According to their scaling relation the true $\Delta G^{*}$ differs from the CNT prediction $\Delta G_{\mathrm{CNT}}^{*}$ by a function only of temperature. It seems that the advanced scaling relations have made an important step towards developing a better phenomenological theory of nucleation to correlate experimental measurements. ${ }^{27}$

\section{E. Comparison with nucleation theorem}

The nucleation theorem ${ }^{28-30}$ states that at a given temperature the variation of the formation free energy of the critical cluster $\Delta G_{i}^{*}$ with the chemical potential difference $\Delta \mu$ between the supersaturated vapor and the bulk liquid is related to the critical cluster size $i^{*}$ by the equation

$$
\frac{d \Delta G_{i}^{*}}{d \Delta \mu}=-i^{*} .
$$

Because this theorem is generic (model independent) it has

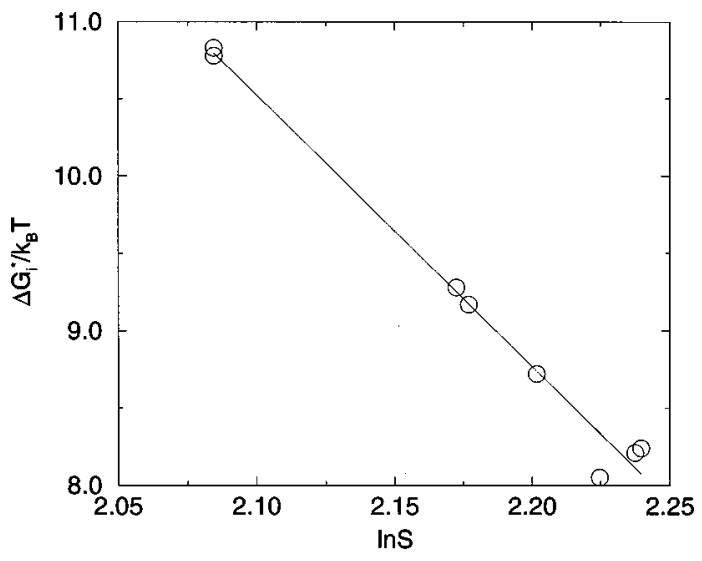

FIG. 9. The $\Delta G_{i}^{*} / k_{B} T$ as a function of $\ln S$ at $T=0.67 \epsilon / k_{B}$. The solid line is a linear fit to the data.

been used in experiments to infer the size of the critical cluster from rates of nucleation. Here, we used Eq. (7) to verify the simulation results.

Since the chemical potential difference $\Delta \mu$ is not available, we substituted a relation for the ideal gas

$$
\Delta \mu=k_{B} T \ln S,
$$

into Eq. (7) and obtained

$$
\frac{d \Delta G_{i}^{*} / k_{B} T}{d \ln S}=-i^{*} .
$$

Figure 9 shows $\Delta G_{i}^{*} / k_{B} T$ as a function of $\ln S$ at $T$ $=0.67 \epsilon / k_{B}$. One can see that $\Delta G_{i}^{*} / k_{B} T$ has a linear relationship with $\ln S$ within the range of supersaturations in our simulation. The solid line in Fig. 9 is a fit to the simulation data and the slope of this line is about -18 . The latter means $i^{*} \sim 18$, which is very close to $i^{*}=20$ (see Table I) obtained from the $\Delta G_{i}^{*}$ versus $i$ curve (see Figs. 2 and 4).

\section{CONCLUSION}

The formation free energies of clusters in supersaturated vapors are obtained by means of a new Monte Carlo method. A key feature of this approach is to impose an upper limit $i_{\text {max }}$ to the cluster size. This cluster size constraint serves as an independent variable to describe the constrained equilibrium states in simulation. First, at given $N, V, T$, we found that a large $i_{\max }$ results in a smaller supersaturation $S$ and a higher formation free energy of critical cluster $\Delta G_{i}^{*}$. The self-consistency of this approach is examined via adjusting the supersaturation from two different routes: varying the overall density $N / V$ or $i_{\max }$. No matter which route is taken to adjust $S$, as long as the final $S$ is the same, the same formation free energy $\Delta G_{i}$ is obtained. Next, we found the calculated formation free energies are independent of the system size $N$ when $N \gg i_{\max }$. Last, we found the calculated critical cluster size not only is consistent with that predicted by Zhukhovitskii who used a quite different simulation method, but also is consistent with that predicted using nucleation theorem.

In this simulation, the (constrained) canonical ensemble was used. However, the constant pressure ensemble should also be applicable to calculate $i^{*}$ and $\Delta G_{i}^{*}$ as long as the 
upper limit of the cluster size $i_{\max }$ is taken. We note that there is another way to apply constraint in the constant pressure ensemble ${ }^{12}$ for calculating $\Delta G_{i}^{*}$. That is to constrain the magnitude of fluctuation of the system volume $V$. In that approach the clusters can grow until their size is limited by the volume of the system $V$. As such, the clusters may interfere with their own periodic images, first pointed out by Duijneveldt and Frenkel. ${ }^{31}$ To what extent this interference affects the calculated free energy barrier height of nucleation requires further study.

We also compared our simulation results with those from the classical nucleation theory. We found CNT overestimates the formation free energy of the critical cluster and underestimates the size at temperatures near the triple point. Our conclusion is consistent with that from the density functional calculation.

In closing, we note that this simulation approach explicitly takes into account both the cluster-vapor interaction and the imperfection of the supersaturated vapor. Thus, this approach can be used to study the carrier-gas effects on vapor-liquid nucleation. ${ }^{32}$ In the future, we will use this approach to examine theoretical works on carrier-gas effects. For example, Kashchiev ${ }^{33}$ found that the second mixed virial coefficient of the gas mixture as well as the second virial coefficient of the carrier gas play important factors in accessing the carrier-gas effects. Research along this direction is underway.

\section{ACKNOWLEDGMENTS}

We are grateful to Dr. G. T. Gao and Dr. K. Koga for useful discussions and Dr. P. R. ten Wolde for sending us his thesis. X.C.Z. thanks the National Science Foundation for support of this work.

${ }^{1}$ D. W. Oxtoby, J. Phys.: Condens. Matter 4, 7627 (1992).

${ }^{2}$ R. Becker and W. Döring, Ann. Phys. (Leipzig) Ser. 5 24, 719 (1935).

${ }^{3}$ F. F. Abraham, Homogeneous Nucleation Theory (Academic, New York, 1974).
${ }^{4}$ Nucleation and Atmospheric Aerosols 1996, edited by M. Kulmala and P. E. Wagner (Pergamon, Oxford, 1996).

${ }^{5}$ A. Laaksonen, V. Talanquer, and D. W. Oxtoby, Annu. Rev. Phys. Chem. 46, 489 (1995).

${ }^{6}$ H. M. Ellerby, C. L. Weakliem, and H. Reiss, J. Chem. Phys. 95, 9209 (1991).

${ }^{7}$ Nucleation, edited by A. C. Zettlemoyer (Marcel Dekker, New York, 1969).

${ }^{8}$ I. Kusaka, Z.-G. Wang, and J. H. Seinfeld, J. Chem. Phys. 108, 3416 (1998).

${ }^{9}$ P. R. ten Wolde, D. Frenkel, and Oxtoby, Phys. Rev. Lett. 81, 3695 (1998).

${ }^{10}$ B. Senger, P. Schaaf, D. S. Corti, R. Bowles, D. Pointu, J.-C. Voegel, and H. Reiss, J. Chem. Phys. (to be published).

${ }^{11}$ W. C. Swope and H. C. Andersen, Phys. Rev. B 41, 7042 (1990)

${ }^{12}$ D. S. Corti and P. Debenedetti, Chem. Eng. Sci. 49, 2717 (1994).

${ }^{13}$ H. Reiss, Ber. Bunsenges. Phys. Chem. 79, 943 (1975).

${ }^{14} \mathrm{H}$. Reiss, Methods of Thermodynamics (Dover, New York, 1965).

${ }^{15}$ M. P. Allen and D. J. Tildesley, Computer Simulation of Liquids (Oxford University Press, New York, 1987).

${ }^{16}$ F. H. Stillinger, J. Chem. Phys. 38, 1486 (1963).

${ }^{17}$ P. Schaaf, B. Senger, and H. Reiss, J. Phys. Chem. B 101, 8740 (1997).

${ }^{18}$ F. F. Abraham and J. A. Barker, J. Chem. Phys. 63, 2266 (1975).

${ }^{19}$ J. K. Johnson, J. A. Zollweg, and K. E. Gubbins, Mol. Phys. 78, 591 (1993).

${ }^{20}$ P. R. ten Wolde, Ph.D. thesis, 1998.

${ }^{21}$ M. Rao, B. J. Berne, and M. H. Kalos, J. Chem. Phys. 68, 1325 (1978).

${ }^{22}$ M. Thompson, K. E. Gubbins, J. P. R. B. Walton, R. A. R. Chantry, and J. S. Rowlinson, J. Chem. Phys. 81, 530 (1984).

${ }^{23}$ D. J. Zhukhovitskii, J. Chem. Phys. 103, 9401 (1995).

${ }^{24}$ X. C. Zeng and D. W. Oxtoby, J. Chem. Phys. 94, 4472 (1991).

${ }^{25}$ C. Hung, M. J. Krasnopoler, and J. L. Katz, J. Chem. Phys. 90, 1856 (1989).

${ }^{26}$ R. McGraw and A. Laaksonen, Phys. Rev. Lett. 76, 2754 (1996).

${ }^{27}$ V. Talanquer, J. Chem. Phys. 106, 9957 (1997).

${ }^{28}$ D. Kashchiev, J. Chem. Phys. 76, 5098 (1982).

${ }^{29}$ Y. Viisanen, R. Strey, and H. Reiss, J. Chem. Phys. 99, 4680 (1993).

${ }^{30}$ D. W. Oxtoby and D. Kashchiev, J. Chem. Phys. 100, 7665 (1994).

${ }^{31}$ J. S. van Duijneveldt and D. Frenkel, J. Chem. Phys. 96, 4655 (1992).

${ }^{32}$ R. H. Heist, M. Janjua, and J. Ahmed, J. Phys. Chem. 98, 4443 (1994); J. A. Fisk and J. L. Katz, J. Chem. Phys. 104, 8649 (1996); D. Kane and M. S. El-Shall, J. Chem. Phys. 105, 7617 (1996); C. C. M. Luijten, K. J. Bosschaart, and M. E. H. Van Dongen, J. Chem. Phys. 106, 8116 (1997).

${ }^{33}$ D. Kashchiev, J. Chem. Phys. 104, 8671 (1996). 